\title{
Telemedicine and Metabolic Diseases in Practice - Weight Predictor Localized for the Czech Population
}

\author{
Ondrej Kade*, Aneta Haskova, Stepan Svacina, Pavla Zurkova, Ondrej Mikes, Kristyna Cmerdova \\ and Martin Matoulek

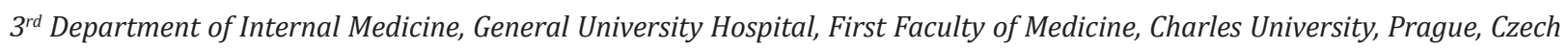 \\ Republic
}

*Corresponding author: Ondrej Kade, $3^{\text {rd }}$ Department of Internal Medicine, General University Hospital, First Faculty of Medicine, Charles University, Prague, Czech Republic

\section{ARTICLE INFO}

Received: 幽 September 09, 2021

Published: 幽 October 05, 2021

Citation: Ondrej Kade, Aneta Haskova, Stepan Svacina, Pavla Zurkova, Ondrej Mikes, et al., Telemedicine and Metabolic Diseases in Practice - Weight Predictor Localized for the Czech Population. Biomed J Sci \& Tech Res 39(2)-2021. BJSTR. MS.ID.006272.

Keywords: Telemedicine, Obesity, Physical activity, Eating habits, Metabolic diseases, Wearables, Weight predictor

\begin{abstract}
We aimed to develop the weight predictor for obese patients. Telemedicine has been gaining more and more importance in recent decades in the supportive treatment of patients with various diseases. In obesitology, the rise of telemedicine occurred at the end of the 20th century, when communication technologies developed and the first diet records appeared via PC, and especially there was a significant increase in the number of obese patients. In diabetology, remote monitoring of blood glucose values using subcutaneous chips with wireless data transmission is being developed. In our research, we involved 248 probands in the monitoring of exercise and eating habits, and on the obtained data we tried to create predictive equations for the development of weight when changing these habits. In developing the prediction equation, we relied on the laboratory model of Dr. Hall, which we adapted for Czech conditions and environment. For adaptation to the Czech environment, we "enriched" Dr. Hall's model with a correction factor that better adapts the model to our conditions.
\end{abstract}

Abbreviations: WHO: World Health Organization; VoIP: Voice over Internet Protocol; CT: Computed Tomography; MRI: Magnetic Resonance Imaging; TCP / IP: Transmission Control Protocol / Internet Protocol; VLCD: Very Low Calorie Diet; BMI: Body Mass Index

\section{Introduction}

Telemedicine has been gaining in importance recently. The term telemedicine is derived from two Greek words - tele ("distance") and medicine (meden = treatment "). According to the European Commission, it is defined as:" Rapid access to shared and remote medical expertise through telecommunications and information technology, regardless of where the patient or relevant information is located. Sometimes the term "telehealth" is used instead of telemedicine [1]. The history of modern telemedicine dates back to the invention of the traditional telephone in the $19^{\text {th }}$ century. At that time, physicians could give information and medical advice by phone. In simple terms, telemedicine can be defined as a supportive medical service using telecommunication technologies [2]. The concept of telemedicine has become established in medical terminology at the instigation of the WHO
(World Heat Organization). Another term used for telemedicine is "distance medicine". However, there are differences in definition within individual fields of telemedicine. The view of individual specializations in telemedicine is inconsistent and may vary. In general, however, the goals of telemedicine can be characterized as follows: to speed up and improve communication between doctors, between doctor and patient, to use modern communication tools and effective information transfer, to improve overall patient care and to improve diagnostic and therapeutic processes [1]. Telemedicine is defined by the WHO as a general term for health activities, services and systems operated remotely through information and communication technologies to promote global health, prevention and health care, as well as education, health care and medical research. The general definition of telemedicine is the 
provision of medical services at a distance, and this term covers a wide range of different applications. There are two basic areas. First, applications that allow real-time monitoring of the patient, eg. in the form of video calls, online transmission of heart rate data, etc. Second, applications that work asynchronously, eg. by storing physiological data (weight, blood pressure, blood glucose, heart rate records from exercise units, etc.) from home monitoring and their subsequent transfer to the database in the medical facility or reconditioning center [3].

The main goal of telemedicine is to provide medical services remotely. Reducing the need for clinical visits is one of the many benefits that telemedicine brings to patients and offers them obvious convenience. Thanks to the use of IT technologies, the patient can receive basic medical care at home. By evaluating the level of medical support provided over the last two to three decades, IT technologies have undoubtedly provided huge benefits to the general public. More powerful, faster computers, and more efficient use of features have allowed more types of services to be extended to more users. For example, several decades ago, a simple request for medical help could only be obtained by searching for a landline at the clinic where the doctor was present. Thanks to the availability of "Voice over Internet Protocol" (VoIP) mobile technology, you can easily pick up your mobile phone and make a video call with your doctor, ie. the doctor providing the consultation does not necessarily have to be present at the clinic. This is just one of many examples where advances in IT technology have contributed to more affordable healthcare [2]. Thanks to the rapid development of technology, telemedicine is currently gaining more and more space and use across medical specializations. In addition, it enables the so-called remote monitoring of the patient, or the data collection from the patient and their subsequent control with professional recommendations with regard to the patient's goals. It is potentially widely used, for example, in diabetology, where it uses subcutaneous sensors in patients with DM, which read the patient's blood glucose level, store data and send it to the physician via a mobile phone connected to the sensor. The physician can evaluate the data and provide feedback to the patient.

The use of telemedicine has common elements in various medical fields. Remote clinical care helps remove barriers and improves access to health services. It is therefore a benefit in urgent and intensive care as well as in the care of chronic patients. Regardless of the medical field in which telemedicine is used, its basic areas of activity can be divided into four categories [1]:
1) Information transfer

2) Remote monitoring

3) Distance therapy

4) Telemedicine education

Telemedicine communication can be further divided into:

1) Voice: The simplest telemedicine service using an analog or digital telephone network. These are, for example, helplines, security lines, consulting and advisory lines.

2) Visual: This includes various imaging methods - teleradiology, CT, MRI, angiography, etc., but also, for example, video conferencing.

3) Data: Data exchange, telemetry, access to databases. It takes place mainly using the Internet and the TCP / IP family of protocols. Includes telemetry - remote monitoring of physiological functions.

\section{Telemedicine in the Treatment of Obesity}

Obesity is one of the civilization diseases, which, together with type 2 diabetes mellitus, has been growing since the 1940s, although it seems to be peaking and the increase is no longer so great. However, according to the latest actual data from 2013, approximately $23 \%$ of the adult population is obese and another $34 \%$ are overweight. Thus, a total of $57 \%$ of the adult population struggles with overweight and obesity (Figure 1) [4]. Obese people are 6 times more likely to get type 2 diabetes. If they exercise regularly, only 3.5 times more often. The development of obesity is undoubtedly due to a decrease in physical activity, and although the increase from 2010 to 2013 appears to have virtually stopped, the time spent on physical activity is still shortening [4]. The only result is a logical decrease in physical ability, which is a key prognostic factor in cardiovascular mortality, especially in old age. The main problem of effective treatment is to obtain quality data and this is the use of telemedicine. Questionnaires, whether related to physical activity or frequency questionnaires, appear to have too large deviations from actual energy intake or expenditure. Although there is no doubt that in a large amount of data, they can provide relatively accurate information about diet and physical activity with quality processing. The validity of the questionnaires on physical activity and their use for epidemiological research has been repeatedly confirmed [5]. However, the individual deviations tend to be large and the recommendations based on the questionnaire survey are not effective. 
Obesity according to BMI index - evolution

All respodents, by years: $\mathrm{n}=3053$ (2000), n=2096 (2005), n=2058 (2008), n=2065 (2010), n=2058 (2013)

Comparison in years 2000, 2005, 2008, 2010, 2013

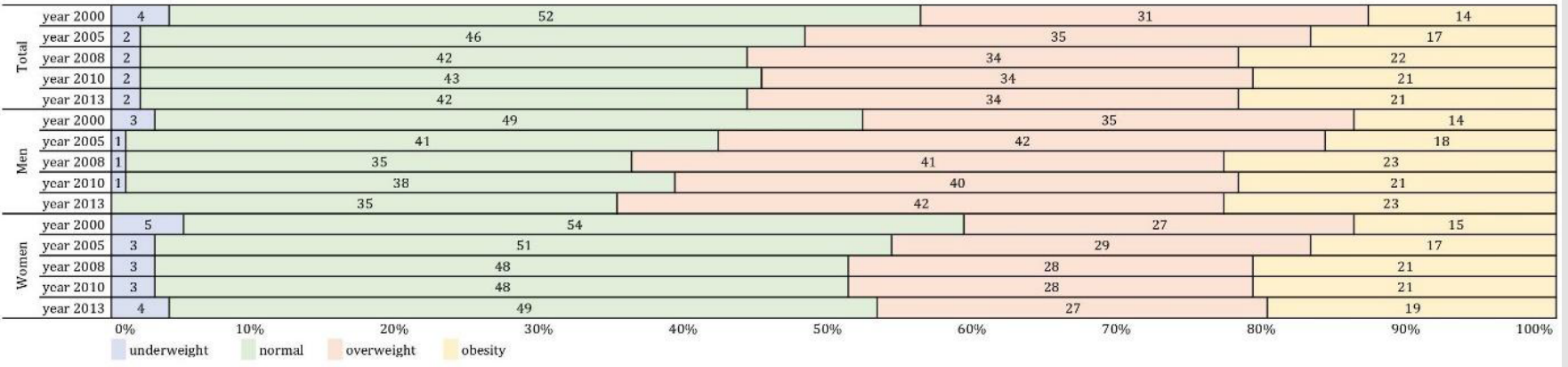

Figure 1: Incidence of obesity in the Czech Republic 2001-2013.

It is not entirely possible to individually calculate the energy value or the proportion of individual components of food in the diet by doctors or nutritional therapists. Various applications are used for this purpose, the quality of applications is often determined by the database that is formed at the time of its creation, but above all by regular updates, which are often greatly underestimated. It is often not even possible to use foreign food databases, because the composition of different foods with similar names is a wellknown problem that is currently being addressed at European Commission level. The specifics of various recipes can be gradually eliminated by entering your own recipes, which quality databases or applications should allow. However, accurate quantity data are much more important for evaluation. This is the only way to get approximately the right result. Immediate recording after a meal is very important. If we record in the evening, even the best applications or food databases will not help us and the error rate will be close to $40 \%$ [4].

Telemedicine procedures will be beneficial especially in the rural population, where there is no good availability of clinical specialists (nutrition therapists, psychologists, etc.). This is also proven by a study by Brown from South Carolina. She says that the key is the availability of care at greater distances for the possibility of regular visits, respectively. interventions. The study showed the same effect of weight reduction in clinical interventions during faceto-face visits as when using telemedicine (videoconferencing, etc.) in the rural population [6]. Similarly, another study demonstrates the effectiveness of smartphone engagement and increased physical activity compared to the control group [7]. There is no doubt about cost-effectiveness in the use of telemedicine, although we have not found a detailed study on this topic. Our unpublished data show that weight loss efficacy increases by approximately $15-20 \%$ when patients use online energy intake and expenditure monitoring. Adherence to the reduction regime is then about 5-6 months longer. According to [8] [9], there are a small number of long-term studies that show that wearables can improve the results of long-term physical activity and weight loss. However, there was insufficient evidence to show a greater benefit compared to the control groups.

Another randomized study [10] shows that the differences between the control group and the group monitored by smartphone were statistically insignificant. However, the remote monitoring group showed better compliance with the regime. The combination of mobile technology with occasional personal interventions appears to be one of the effective tools in the clinical treatment of obesity. Obesitology is a medical field in which the effective application of distance communication is possible. Self-monitoring, lifestyle change, diet and physical activity are absolutely essential in a reduction mode. Professional medical supervision is possible via information and telecommunication technologies, via the Internet or via smartphones. The advantage of the previous is the possibility of regular communication, whether synchronous or asynchronous. In this context, modern technologies have great potential to help fight obesity, especially in young patients. However, recent study results show that information technologies are not fully utilized in treatment. In the Czech Republic, where the research was conducted, it is not possible to look for barriers only on the part of the patient, because it is not quite common practice on the part of the doctor to use online communication and data sharing in this way. However, for physicians, continuous monitoring of patient outcomes can mean faster detection of patients' health risks [11].

The advantages of telemedicine in obesitology include monitoring patients, reducing the time required for hospitalization, reducing waiting times for personal examinations, streamlining consultations and improving the overall quality of care provided $[11,12]$. As mentioned above, obesitology mainly uses patient selfmonitoring, in particular the control of dietary records, physical activity and other monitored data such as glycaemia, blood pressure, weight, etc. The main advantage of telemedicine is to support patient motivation and improve treatment cooperation, and not only in obese or overweight patients. In terrain we can 
use modern technologies such as pedometers, smart bracelets or watches, heart rate sensors, glucometers, blood pressure monitors, scales (more expensive scales can measure the bioimpedance method of body composition, i.e. the amount of fat, muscle, water in the body) that are connected to a mobile phone (usually via Bluetooth) or online questionnaire completion (nutritional or psychological). Eating habits are monitored online (web or mobile application) on a website with a large food database. The most used application in our country with the widest and regularly updated database is the portal www.kaloricketabulky.cz. The application is free and can be used both in web form and in a mobile phone or tablet as an application. To summarize the above, telemedicine has an important role in obesitology and its use consists mainly in (self) monitoring of patients and subsequent data processing, their evaluation and rapid feedback without the acute need for personal contact.

\section{Materials and Methods}

As part of improving patient care and simplifying cooperation with the patient in obesitology, the "Weight Predictor" portal for the Czech population is being developed. The main goal of this portal is, based on data obtained from the patient, to predict the development of his weight. The purpose of the predictor is to learn from quality data and on the basis of this data and to create a so-called artificial neural network that will be able to respond to changes in the "behavior" of the patient.

\section{At the Moment, the Predictor has Completed the $\mathbf{1}^{\text {st }}$ Phase of Data Collection. The Following Data were Collected from Patients (Figure 2)}

1) Energy Intake: Obtained from the applications caloricketabulky.cz, MyFitnessPal and Nutridata.cz

2) Energy Expenditure: Data obtained from smart devices Garmin (Figure 3), Fitbit or others connectable to Google Fit or Apple Health in a mobile phone

3) Questionnaires:

a. Lifestyle questionnaire

b. Psychological profile

c. Lifetime history of weight

d. Anamnestic questionnaire

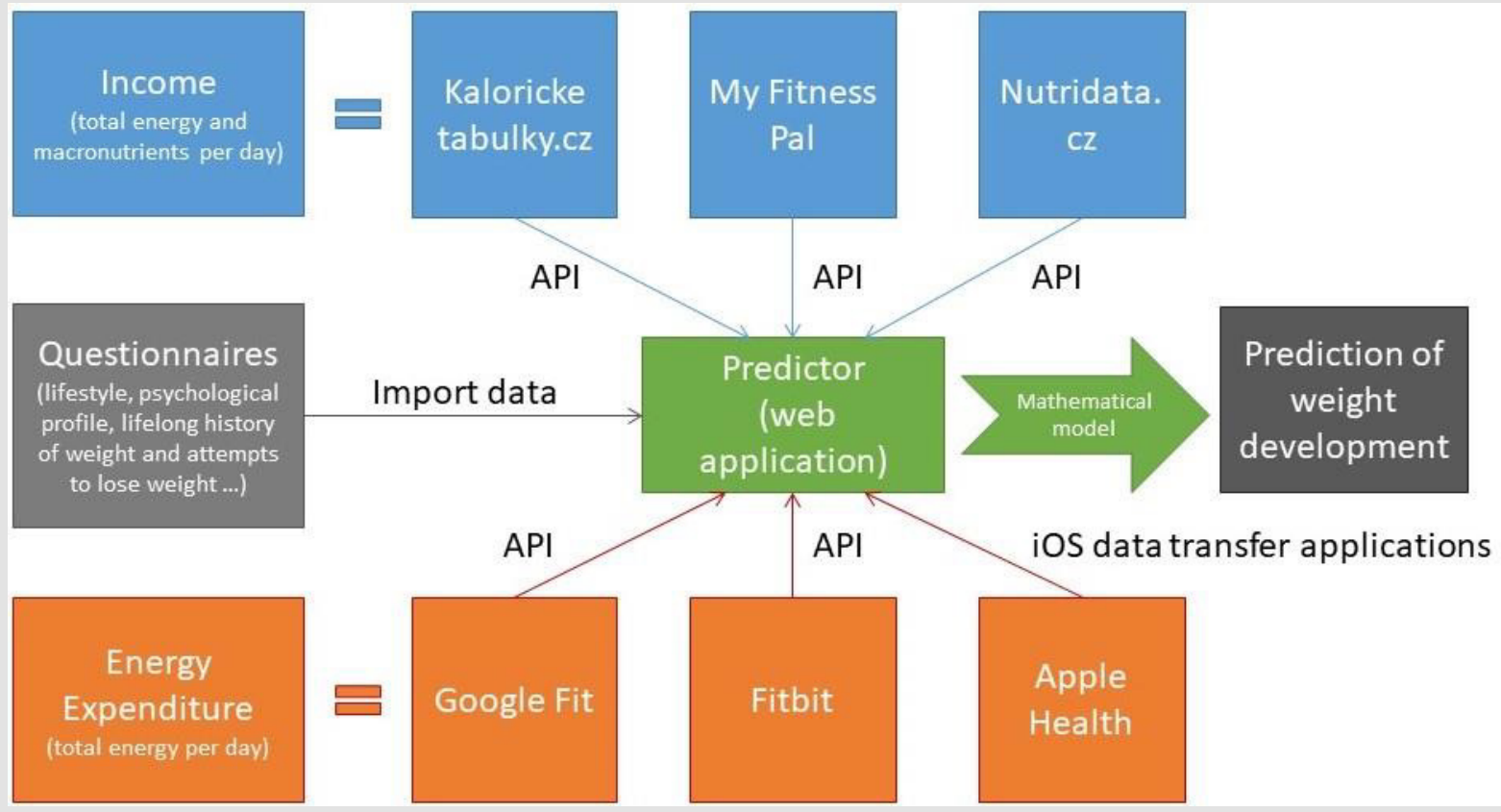

Figure 2: Weight Predictor Data Collection Scheme (Energy intake using the online platform kaloricketabulky.cz, myfitnesspal. com or nutridata.cz. Most often used kaloricketabulky.cz. Energy expenditure - monitored habitual activity (steps) using bracelets with a pedometer via GoogleFit, Fitbit AppleHealth or Garmin. Furthermore, data were collected from questionnaires). 
The Data Collection Methodology Included a Total of 6 Weeks of Patient Monitoring with thorough Control of Data Collection. The Data Collection Schedule was as follows

1) Filling in the form - control of the meeting the criteria for inclusion in the program

2) Boot regime

a. Explanation of the program in details

b. Setting up one of the monitoring systems for energy intake and output - see Figure 1.

c. Body composition measurement

d. Ordering a placement visit

3) Placement visit

a. After 1-2 weeks from the first visit

b. Thorough compliance check:

i. Recording the weight, filling in the energy intake and expenditure (steps)

ii. Weighing and measuring body composition

iii. Completion of anamnestic questionnaire

iv. Completion of a psychological questionnaire

v. Record of lifelong weight development

4) Controls

a. A personal visit with consultation and measurement of body composition each week

b. 2 online control of data collection in a week

c. Possibility of online consultation

5) Final Visit

a. Weighing and measuring body composition

b. Quality control of data and data sending

c. In case of a successful regime, it was possible to extend to the second successor regime and possibly to the third

\section{Results}

A total of 1193 applicants were registered in the predictor, but unfortunately there was a very large decrease of applicants, so only 248 programs were successfully completed. An overview of data collection participants is shown in Tables $1 \mathrm{~A}-1 \mathrm{C}$ and Figures 4A-4E. The biggest problems in data acquisition were mainly the technological barrier in the elderly population (70+) and also less interest in the male population. The best response was in the 40-50 age group in both sexes. From the above, however, it is clear that the obtained data will not be able to "train" the neural network. The "success" of completed programs is shown in Tables 2A \& 2B. It can be seen from Table 2 that out of 1193 applicants, only 248 completed, which is less than $21 \%$ of all participants involved. In the beginning, most of the applicants were motivated, but over time, the decrease of probands was great. 203 participants did not complete the program due to time constraints, 386 did not fill in calorie tables or weight, 306 probands had insufficiently filled in data during data collection and therefore it was not possible to use the data. 53 probands did not complete due to health reasons. The biggest problems in data acquisition were mainly the technological barrier in the elderly population (70+) and also less interest in the male population. The best response was in the 40-50 age group in both sexes. From the above, however, it is clear that the obtained data will not be able to "train" the neural network. The "success" of completed programs is shown in Tables 2A \& 2B. It can be seen from Table 2 that out of 1193 applicants, only 248 completed, which is less than $21 \%$ of all participants involved. In the beginning, most of the applicants were motivated, but over time, the decrease of probands was great. 203 participants did not complete the program due to time constraints, 386 did not fill in calorie tables or weight, 306 probands had insufficiently filled in data during data collection and therefore it was not possible to use the data. 53 probands did not complete due to health reasons. The calculated correction coefficients are shown in Tables 3A-3C, both for the whole set and even with the division into men and women. As can be seen in Table 3 , the correction factor is on average comparable for both sexes and is around 5.5. Which is a relatively large number (Figures 5 \& 6).

Table 1: Set of probands:

\begin{tabular}{|c|c|c|c|c|c|}
\hline$A-\operatorname{ALL}(n=248)$ & Age & Height (kg) & Weight (kg) & BMI $\left(\mathrm{kg} / \mathrm{m}^{2}\right)$ & $\begin{array}{l}\text { Waist circumference } \\
\text { (cm) }\end{array}$ \\
\hline Mean & 46,94 & 170,62 & 96,75 & 58,78 & 106,98 \\
\hline Median & 46,00 & 170,00 & 95,00 & 58,82 & 107,00 \\
\hline Minimum & 18,00 & 150,00 & 50,00 & 50,00 & 69,00 \\
\hline Maximum & 76,00 & 200,00 & 222,00 & 66,67 & 180,00 \\
\hline Standard deviation & 11,98 & 9,39 & 24,72 & 3,15 & 17,69 \\
\hline Dispersion & 143,43 & 88,08 & 610,99 & 9,94 & 313,05 \\
\hline
\end{tabular}




\begin{tabular}{|c|c|c|c|c|c|}
\hline B - Men (n = 70) & Age & Height (kg) & Weight (kg) & BMI (kg/m2) & $\begin{array}{c}\text { Waist circumference } \\
\text { (cm) }\end{array}$ \\
\hline Mean & 47,54 & 181,14 & 113,83 & 55,31 & 117,19 \\
\hline Median & 46,00 & 181,00 & 109,75 & 55,25 & 116,50 \\
\hline Minimum & 18,00 & 168,00 & 76,00 & 50,00 & 89,00 \\
\hline Maximum & 72,00 & 200,00 & 222,00 & 59,52 & 180,00 \\
\hline Standard deviation & 11,45 & 8,02 & 22,99 & 5,42 & 16,63 \\
\hline Dispersion & 131,06 & 64,36 & 528,42 & BMI (kg/m2) & Waist circumference \\
(cm)
\end{tabular}

A. General overview of probands who completed the program and had sufficiently written or transferred data,

B. Set of men,

C. Set of women.

Table 2: Overview of completed programs

\begin{tabular}{|c|c|c|c|c|c|c|c|c|c|}
\hline \multirow{2}{*}{$\begin{array}{l}\text { Percentage } \\
\text { of } \\
\text { completion }\end{array}$} & \multicolumn{3}{|c|}{ All } & \multicolumn{3}{|c|}{ Men } & \multicolumn{3}{|c|}{ Women } \\
\hline & Demand & Involved & Completed & Demand & Involved & Completed & Demand & Involved & Completed \\
\hline 18 - 29 у.о. & $100 \%$ & $229 \%$ & $26 \%$ & $100 \%$ & $132 \%$ & $9 \%$ & $100 \%$ & $341 \%$ & $49 \%$ \\
\hline 30 - 39 у.о. & $100 \%$ & $259 \%$ & $47 \%$ & $100 \%$ & $182 \%$ & $22 \%$ & $100 \%$ & $346 \%$ & $78 \%$ \\
\hline 40 - 49 у.о. & $100 \%$ & $282 \%$ & $74 \%$ & $100 \%$ & $223 \%$ & $51 \%$ & $100 \%$ & $337 \%$ & $92 \%$ \\
\hline 50 - 59 у.о. & $100 \%$ & $236 \%$ & $57 \%$ & $100 \%$ & $159 \%$ & $28 \%$ & $100 \%$ & $314 \%$ & $87 \%$ \\
\hline 60 - 69 у.о. & $100 \%$ & $169 \%$ & $43 \%$ & $100 \%$ & $152 \%$ & $34 \%$ & $100 \%$ & $182 \%$ & $48 \%$ \\
\hline 70 and over & $100 \%$ & $63 \%$ & $7 \%$ & $100 \%$ & $91 \%$ & $4 \%$ & $100 \%$ & $38 \%$ & $12 \%$ \\
\hline Overall & $100 \%$ & $217 \%$ & $45 \%$ & $100 \%$ & $161 \%$ & $25 \%$ & $100 \%$ & $273 \%$ & $65 \%$ \\
\hline \multirow{2}{*}{$\begin{array}{c}\text { Completed } \\
\text { programs }\end{array}$} & \multicolumn{3}{|c|}{ All } & \multicolumn{3}{|c|}{ Men } & \multicolumn{3}{|c|}{ Women } \\
\hline & Demand & Involved & Completed & Demand & Involved & Completed & Demand & Involved & Completed \\
\hline 18 - 29 у.о. & 95 & 218 & 25 & 51 & 67 & 5 & 44 & 150 & 22 \\
\hline 30 - 39 у.о. & 102 & 264 & 48 & 54 & 98 & 12 & 48 & 166 & 37 \\
\hline 40 - 49 у.о. & 100 & 282 & 74 & 48 & 107 & 24 & 52 & 175 & 48 \\
\hline 50 - 59 у.о. & 102 & 241 & 58 & 51 & 81 & 14 & 51 & 160 & 44 \\
\hline 60 - 69 у.о. & 88 & 149 & 38 & 41 & 62 & 14 & 47 & 86 & 23 \\
\hline 70 and over & 63 & 40 & 5 & 30 & 27 & 1 & 33 & 13 & 4 \\
\hline Overall & 550 & 1193 & 248 & 275 & 443 & 70 & 275 & 750 & 178 \\
\hline
\end{tabular}

A. Percentage overview of completed programs,

B. Absolute numbers of involved and completed programs according to age categories. 
Table 3.

\begin{tabular}{|c|c|c|c|}
\hline \multicolumn{4}{|c|}{ Correction Coefficients for a given Set of Probands } \\
\hline & Error on the Test Set (kg) & Error on the Training Set (kg) & Value of the Correction Factor \\
\hline Number of probands & 256 & 256 & 256 \\
\hline Mean & 1,01 & 0,44 & 5,55 \\
\hline Standard deviation & 0,66 & 0,30 & 1,81 \\
\hline Minimum & 0,27 & 0,13 & 2,54 \\
\hline Maximum & 3,77 & 2,22 & 10,00 \\
\hline \multicolumn{4}{|c|}{ Correction Coefficients for a given Set of Men } \\
\hline & Error on the Test Set (kg) & Error on the Training Set (kg) & Value of the Correction Factor \\
\hline Number of probands & 73 & 73 & 73 \\
\hline Mean & 1,31 & 0,51 & 5,54 \\
\hline Standard deviation & 0,79 & 0,20 & 2,04 \\
\hline Minimum & 0,39 & 0,17 & 2,54 \\
\hline Maximum & 3,77 & 0,99 & 10,00 \\
\hline \multicolumn{4}{|c|}{ Correction Coefficients for a given Set of Women } \\
\hline & Error on the Test Set (kg) & Error on the Training Set (kg) & Value of the Correction Factor \\
\hline Number of probands & 183 & 183 & 183 \\
\hline Mean & 0,89 & 0,42 & 5,56 \\
\hline Standard deviation & 0,56 & 0,33 & 1,72 \\
\hline Minimum & 0,27 & 0,13 & 2,83 \\
\hline Maximum & 3,28 & 2,22 & 10,00 \\
\hline
\end{tabular}

A. Total values of correction coefficients for a group of probands,

B. Calculated correction coefficients for a set of men,

C. Calculated correction coefficients for a set of women.

Figure 3: Garmin device for steps collection. 

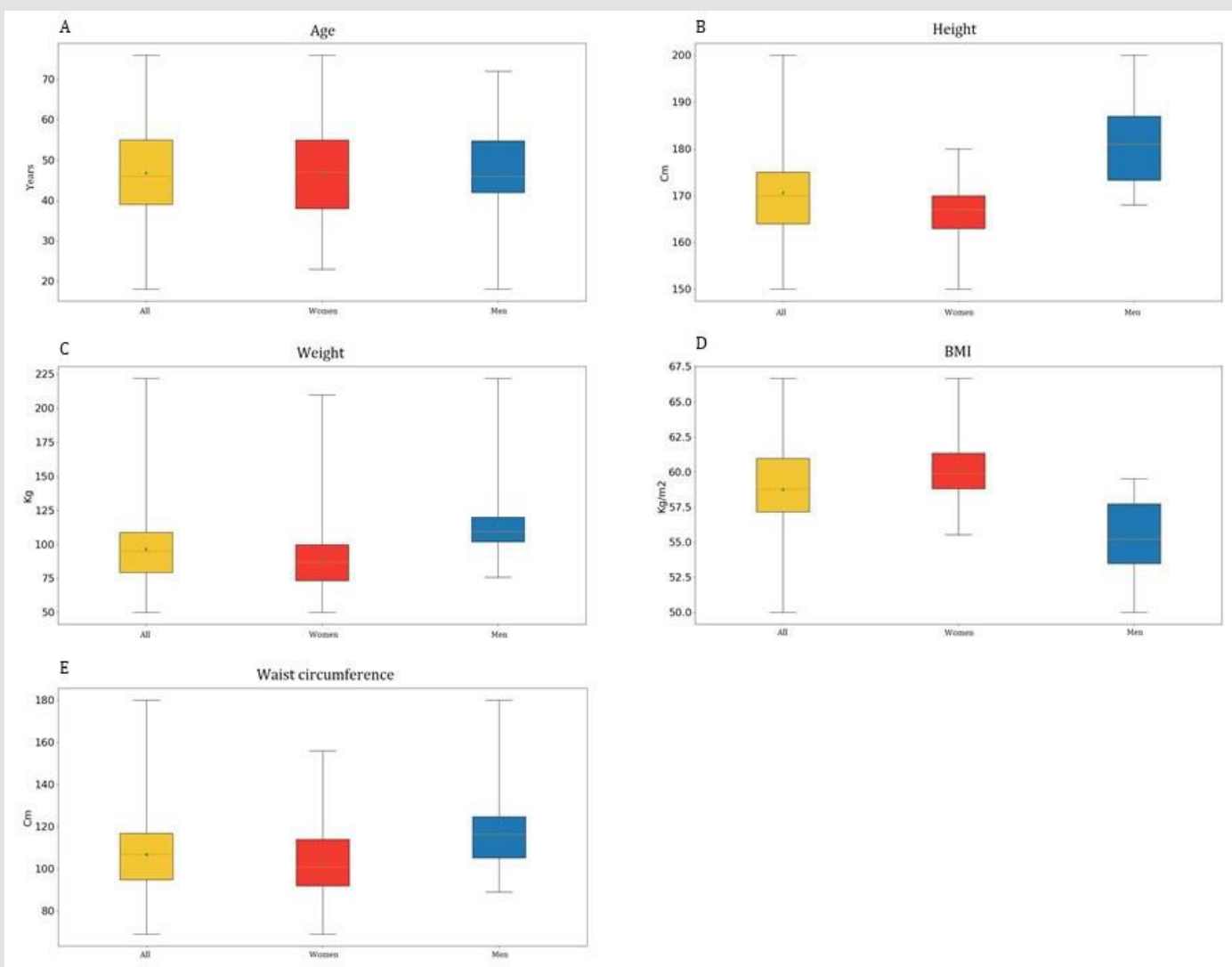

Figure 4: Box plots for:
A. A age,
B. Height,
C. Weight,
D. BMI,
E. Waist circumference

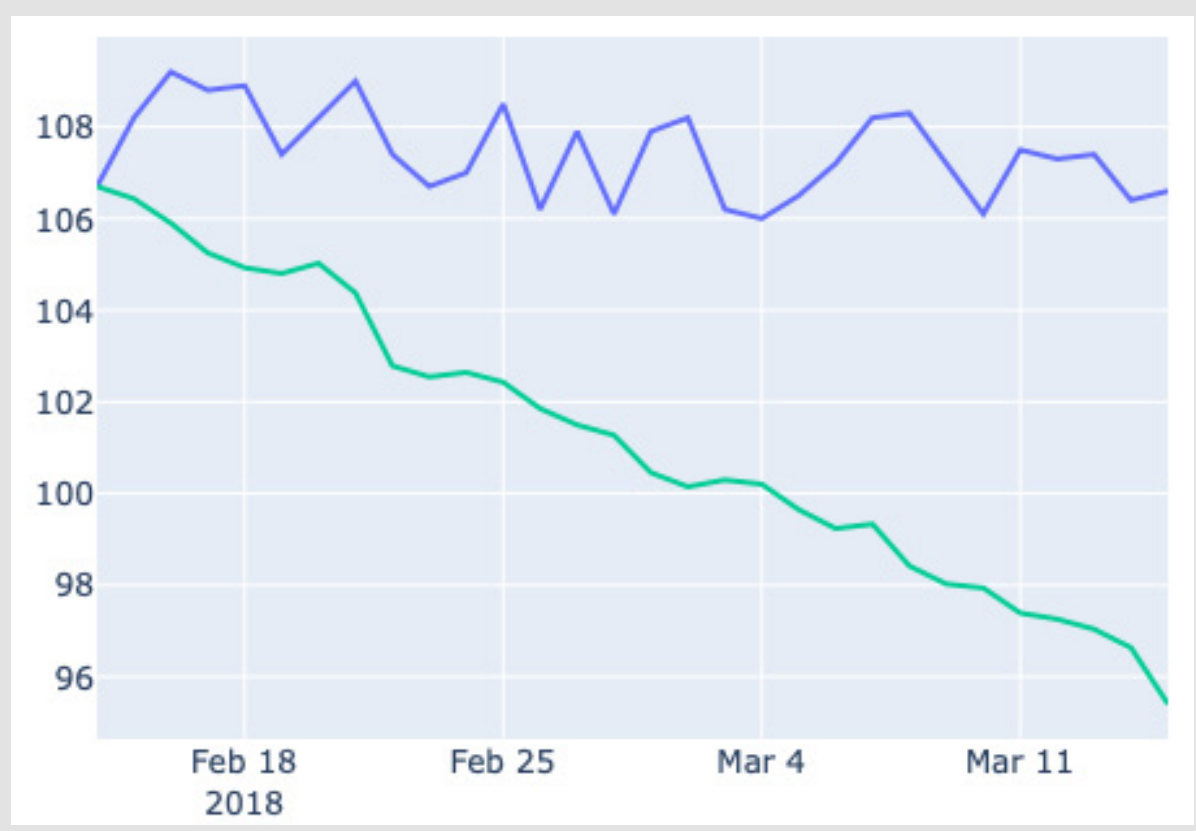

Figure 5: Demonstration of the development of the real weight of a random proband (blue color) and predicted development according to the equation of $\mathrm{dr}$. Hall (green color) in time. 


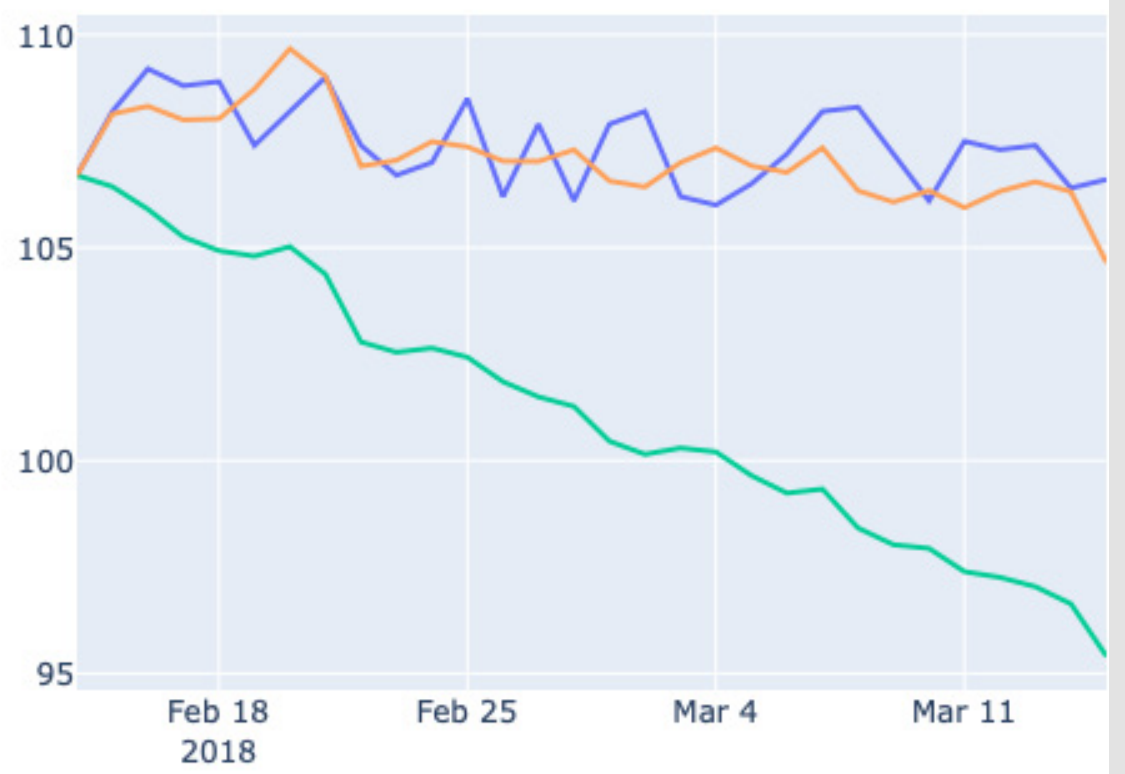

Figure 6: Demonstration of the development of the real weight of a random proband (blue color) and predicted development according to the equation of dr. Hall (green color) in time.

\section{Discussion}

Telemedicine certainly has a place in obesity and its importance will undoubtedly take on. In addition to monitoring applications and systems, we tried to create software for predicting the weight of Czech patients based on their eating and exercise habits. Based on the collected data, it was not possible to build a neural network that would learn from its data and be able to predict the development of the weight of the individual. The amount of data to create the model was not enough. We therefore used the dynamic-mathematical model of Dr. Hall [13] as a starting model for prediction. as a basis for calculating the prediction. Due to the development of this model on laboratory data, it could not be fully utilized for our purposes. The question is what is the cause of the big difference in the prediction of the used model and real data in our probands. Our probands collected data by self-collection and therefore the collected data could be distorted. No large variations were observed in mostly middle-aged women. There were variations in men's diet, we called them "score lies." A possible explanation is that men tend to underestimate portions (they are lazy to weigh). One possible explanation is that a "normal" portion entered in caloric tables does not normally satisfy a man, which is related to the previous one. When a man enters a regular portion in caloric tables, but does not weigh the food, he will increase his income (compared to caloric tables), because his normal portion according to caloric tables exceeds its caloric value. Given the above, we calculated and applied a correction factor to our data, which eliminated the deviations and after incorporating the correction factor, the model showed more significant results for our population [14].
To further refine the model, it would be necessary to test other parameters of the model such as output, content of elements and vitamins in the diet of the Czech population and it was appropriate to test the model on data where the diet remains the same throughout the measurement. The next step in the development of the model should also be the implementation of glycaemia and their influence on the development of body weight, which will be the result of our further work.

\section{Funding}

The study was supported by the Ministry of Health, Czech Republic, RVO-VFN 64165 and by the Charles University in Prague, project GA UK No. 316120 and Progres Q25.

\section{Conflicts of Interest}

All authors have agreed with the content of the manuscript. There is no conflict of interest between the authors.

\section{References}

1. Streda, Leos A Karel Hana (2016) E Health a telemedicína: učebnice pro vysoké školy. In: Streda, Leos A Karel Hana (Eds.)., Praha: Grada Publishing, Czech Republic.

2. Fong Bernard A CM, Fong AC K LI (2020) Telemedicine technologies: information technologies in medicine and digital health $\left(2^{\text {nd }} E d n.\right)$., In: Fong Bernard A CM, Fong AC K LI (Eds.)., Hoboken: Wiley, USA.

3. Tuka Vladimir a kol (2018) Preventivní kardiologie pro praxi. In: Tuka Vladimir a kol (Edt.)., Praha: NOL-nakladatelství odborné literatury.

4. M Matoulek, S Svacina, J Lajka (2010) Výskyt obezity a jejich komplikací. Vnitř Lék 56(10): 1019-1027. 
5. Wolf AM, Hunter DJ, Colditz GA, JE Manson, MJ Stampfer, et al. (1994) Reproducibility and validity of a self-administered physical activity questionnaire. Int J Epidemiol 23(5): 991-999.

6. Brown JD, Hales S, Evans TE, Turner T, Sword DO, et al. (2018) Description, utilisation and results from a telehealth primary care weight management intervention for adults with obesity in South Carolina. J Telemed Telecare 26(1-2): 1357633X18789562.

7. Lee $\mathrm{CH}$, Cheung B, Yi GH, Oh B, Oh YH (2018) Mobile health, physica activity, and obesity: Subanalysis of a randomized controlled trial. Medicine (Baltimore) 97(38): e12309.

8. Pelosi P, Gregoretti C (2010) Perioperative management of obese patients. Best Pract Res Clin Anaesthesiol 24(2): 211-225.

9. Fawcett E, Van Velthoven MH, Meinert E (2020) Long-Term Weight Management Using Wearable Technology in Overweight and Obese Adults: Systematic Review. JMIR Mhealth Uhealth 8(3): e13461.

\section{ISSN: 2574-1241}

DOI: $10.26717 /$ BJSTR.2021.39.006272

Ondrej Kade. Biomed J Sci \& Tech Res

(C) (P) This work is licensed under Creative

Submission Link: https://biomedres.us/submit-manuscript.php
10. Thomas JG, Bond DS, Raynor HA, Papandonatos GD, Wing RR (2019) Comparison of Smartphone-Based Behavioral Obesity Treatment with Gold Standard Group Treatment and Control: A Randomized Trial. Obesity 27(4): 572-580.

11. Maresova P, Hruska J (2020) Management of Overweight and Obesity: Technology-Based Interventions Among Generation Y. Smart Homecare Technology and TeleHealth 7: 1-8.

12. Park HS, Park CY, Oh SW, Yoo HJ (2008) Prevalence of obesity and metabolic syndrome in Korean adults. Obes Rev 9(2): 104-107.

13. Hall KD, Sacks G, Chandramohan D, Carson C Chow, Y Claire Wang, et al (2011) Quantification of the effect of energy imbalance on bodyweight. Lancet 378(9793): 826-837.

14. Guo J, Brager DC, Hall KD (2018) Simulating long-term human weightloss dynamics in response to calorie restriction. Am J Clin Nutr 107(4): 558-565.

BIOMEDICAL
RESEARCHES $\quad \begin{aligned} & \text { Assets of Publishing with us } \\ & \text { - Global archiving of articles }\end{aligned}$

